\title{
Borel 型概率计量逻辑
}

\author{
周红军*, 王国俊 \\ 陕西师范大学数学与信息科学学院, 西安 710062 \\ * 通信作者. E-mail: hjzhou@snnu.edu.cn, sdzhjun@gmail.com \\ 收稿日期: 2008-12-02; 接受日期: 2010-10-08 \\ 国家自然科学基金 (批准号: 61005046, 10771129)、陕西省自然科学基础研究计划 (批准号: 2010JQ8020) 和中央高校基本科研业 \\ 务费专项资金 (批准号: GK200902048) 资助项目
}

\begin{abstract}
摘要 视全体赋值之集为通常乘积拓扑空间, 利用该空间上的 Borel 概率测度在二值命题逻辑中引 入了公式的概率真度概念. 该方法既克服了计量逻辑学要求赋值集上的概率测度必须为均匀概率测 度的无穷可数乘积的局限, 又弥补了概率逻辑学只讲局部而缺乏整体性的不足; 证明了计量逻辑学 中公式的真度、随机真度以及概率逻辑学中公式的概率等概念都可作为本文提出的概率真度的特例 而纳入到统一的框架中, 从而实现了计量逻辑学与概率逻辑学的融合与统一; 证明了逻辑闭理论与 赋值空间中的拓扑闭集是- - 对应的以及概率真度函数与赋值空间上的 Borel 概率测度是一样多的 等若干结论; 本文的第 4 节给出了公式的概率真度的公理化定义, 证明了公式集上满足 Kolmogorov 公理的任一 $[0,1]$ 值函数均可由赋值空间上的某 Borel 概率测度按本文的方法所表出, 从而建立了二 值命题逻辑框架下的概率计量逻辑的理论体系.
\end{abstract}

关键词 概率真度 有限分离性质 概率逻辑 计量逻辑 概率计量逻辑

\section{1 引言}

为使逻辑推理能够处理不确定性的概念和信息, 以更好地符合人脑的思维模式和推理方法, 文献 [1] 在 1984 年通过把概率的思想引入到二值命题逻辑中定义了逻辑公式的概率概念, 以此建立了概率 逻辑学. 随后文献 [2] 也独立地提出了这一思想, 文献 $[3,4]$ 对这一理论作了系统总结. 在概率逻辑学 中, 一个公式的概率是由它的状态描述 (state-description) 集上的概率分布所唯一决定的. 当给定不同 的概率分布时就得到该公式的不同的概率, 这固然很好地反映了概念和信息的不确定性, 但一个公式 的状态描述总是有限个的并且不同公式的状态描述也未必相同, 所以概率逻辑学中公式的概率只是针 对具体公式而言的, 不同公式的概率也因此没有了可比性. 概率逻辑学中的基本理论也是针对具体的 有效推理展开的, 这个有效推理也只涉及有限多个公式, 而二值命题逻辑中的公式却是无限多的, 所 以就从概率逻辑学理论的整体性上看, 似乎只具有局部性而缺乏整体性.

另一方面, 文献 [5] 利用势为 2 的均匀概率测度空间的无穷可数乘积在二值命题逻辑中从整体上 引入了公式的真度概念, 随后便引发了一系列相关的研究 (参见文献 [6-12]). 文献 [13,14] 对这方面的 研究成果作了整理和总结, 形成了计量逻辑学. 计量逻辑学一方面具有了整体性的优点, 同时又有缺 少随机性的不足. 事实上, 在计量逻辑学中每个公式都被赋予了一个真度, 但在该真度意义下, 每个原 
子公式都有相同的真度 $\frac{1}{2}$, 而且任意两个不同原子公式的合取的真度恰等于这两个原子公式的真度的 乘积. 用概率的观点来考察, 即每个原子公式为真的概率均为 $\frac{1}{2}$, 并且它们相互独立. 这种把原子公式 为真的概率等同看待并且要求独立的观点似乎与现实世界中各种简单命题成立的概率不尽相同, 也未 必独立的事实相悖. 所以赋予不同原子公式不同的概率可以使由此产生的公式更具实用性. 基于这样 的考虑, 文献 [15] 利用 $(0,1)$ 上的随机数列在二值命题逻辑中提出了公式的随机真度的概念, 从一定 程度上弥补了计量逻辑学缺乏随机性的不足. 但它仍要求原子公式彼此独立, 其根源在于每个随机数 列都唯一地生成了整个赋值集上的一个乘积概率测度 (参见文中 (6) 式), 这种生成方式就决定了其独 立性. 正如前面所说, 现实生活中大量实际命题都不是独立的, 对于这一问题文献 [3] 也专门独设章节 进行了讨论. 那么能否同时取概率逻辑学和计量逻辑学的优点而又能弥补它们各自的不足从而使它们 达到融合与统一呢?

本文正是以上述问题为出发点, 利用赋值空间上的 Borel 概率测度在二值命题逻辑中引入公式的 概率真度的概念, 进而把计量逻辑学中公式的真度、随机真度以及概率逻辑学中公式的概率都作为特 例纳入到统一的框架中. 这样既克服了计量逻辑学要求赋值集上的概率测度必须为均匀概率测度的无 穷可数乘积的局限, 又弥补了概率逻辑学缺乏整体性的不足, 同时也保留了它们各自的优点, 从而实 现这两个学科间的融合. 所以计量逻辑学和概率逻辑学中的所有基本理论都可在本文的框架下进行讨 论. 本文还将走的更远, 第 4 节将利用概率学中的 Kolmogorov 公理 ${ }^{[16]}$ 给出公式的概率真度的公理 化定义及其若干等价刻画条件, 并最终证明公式集上满足 Kolmogorov 公理的任一 $[0,1]$ 值函数都可 由赋值空间上的唯一 Borel 概率测度按本文的方法所表出, 从而建立二值命题逻辑框架下的概率计量 逻辑理论.

\section{2 预备知识}

本节只扼要地回忆概率逻辑学以及计量逻辑学中的基本概念和结论. 有关二值命题逻辑的基本知 识, 请见文献 [14,17], 且本文沿用文献 [14] 中的符号, 如, 用 $\Omega=\{0,1\}^{\omega}$ 表示全体赋值之集; 用 $F(S)$ 表示全体公式之集, 其中 $S=\left\{p_{1}, p_{2}, \ldots\right\}$ 表示全体原子公式之集.

在介绍概率逻辑学中公式的概率之前, 先介绍 “状态描述” 这一概念.

定义 2.1 ${ }^{[1]}$ 设 $A=A\left(p_{1}, \ldots, p_{n}\right)$ 是由原子公式 $p_{1}, \ldots, p_{n}$ 构成的公式, 规定 $p_{i}^{1}=p_{i}, p_{i}^{0}=\neg p_{i}$. 设 $\left(x_{1}, \ldots, x_{n}\right) \in\{0,1\}^{n}$, 则称公式 $p_{1}^{x_{1}} \wedge \cdots \wedge p_{n}^{x_{n}}$ 为 $A$ 的一个状态描述.

易见公式 $A=A\left(p_{1}, \ldots, p_{n}\right)$ 共有 $2^{n}$ 个不同的状态描述, 记为 $S_{1}, \ldots, S_{2^{n}}$.

定义 $2.2^{[1]}$ 设 $P$ 是公式 $A=A\left(p_{1}, \ldots, p_{n}\right)$ 的状态描述集 $\left\{S_{1}, \ldots, S_{2^{n}}\right\}$ 上的概率分布, 则 $A$ 的 概率 $P(A)$ 定义为

$$
P(A)=\sum\left\{P\left(\left\{S_{i}\right\}\right) \mid A \text { 在状态 } S_{i} \text { 下为真 }\right\},
$$

这里 “ $A$ 在状态 $S_{i}$ 下为真” 是指当 $v\left(S_{i}\right)=1$ 时 $v(A)=1, v \in \Omega$.

由状态描述的定义知, 状态描述 $p_{1}^{x_{1}} \wedge \cdots \wedge p_{n}^{x_{n}}$ 与 $n$ 维 $0-1$ 向量 $\left(x_{1}, \ldots, x_{n}\right)$ 唯一对应. 为简单 起见, 我们直接把 $\left(x_{1}, \ldots, x_{n}\right)$ 称为 $A$ 的一个状态描述. 此时状态描述集上的概率分布 $P$ 就转化为 $\{0,1\}^{n}$ 上的概率分布了, $A$ 在状态 $\left(x_{1}, \ldots, x_{n}\right)$ 下为真就是指 $\bar{A}\left(x_{1}, \ldots, x_{n}\right)=1$, 这里 $\bar{A}$ 是 $A$ 诱导的 Boole 函数. 所以 (1) 式可化为

$$
P(A)=\sum\left\{P\left(\left\{\left(x_{1}, \ldots, x_{n}\right)\right\}\right) \mid\left(x_{1}, \ldots, x_{n}\right) \in \bar{A}^{-1}(1)\right\}=P\left(\bar{A}^{-1}(1)\right) .
$$


例 2.3 (i) 设 $A=p_{1} \vee p_{2}$, 则 $A$ 有 4 个状态描述 $(1,1),(1,0),(0,1),(0,0)$. 设 $P(\{(1,1)\})=$ $0.1, P(\{(1,0)\})=0.6, P(\{(0,1)\})=0.2, P(\{(0,0)\})=0.1$ (简记为 $P=(0.1,0.6,0.2,0.1))$, 则 $P$ 是状态描 述集 $\{0,1\}^{2}$ 上的一个概率分布, $P(A)=P\left(\bar{A}^{-1}(1)\right)=P(\{(1,1),(1,0),(0,1)\})=0.1+0.6+0.2=0.9$. 若 $P=(0.25,0.25,0.25,0.25)$, 则 $P(A)=0.25 \times 3=0.75$.

(ii) 设 $B=p_{1}$, 则 $B$ 有两个状态描述 $(1),(0)$. 设 $P=(0.8,0.2)$ 是 $\{0,1\}$ 上的一个概率分布, 则 $P(B)=P\left(\bar{B}^{-1}(1)\right)=P(\{(1)\})=0.8$.

虽然 0.9 与 0.8 作为数字是可以比较的, 但当它们分别作为公式 $A$ 和 $B$ 的概率时二者就没有了 可比性, 这是因为 $A$ 和 $B$ 的状态描述集不同, 其上的概率分布自然也不同. 这不能不说是概率逻辑学 的一个缺憾.

计量逻辑学利用势为 2 的均匀概率测度空间的无穷可数乘积在二值命题逻辑中从整体上引入了 公式的真度, 从而也弥补了概率逻辑学中概率分布只定义在有限集合上致使公式的概率没有了可比性 的缺憾.

定义 $2.4^{[5]}$ 设 $X_{m}=\{0,1\}, \mu_{m}$ 是 $X_{m}$ 上的均匀概率测度, 令 $X=\prod_{m=1}^{\infty} X_{m}$ (即 $\left.X=\Omega\right), \mathscr{A}$ 是 由 $X$ 中全体形如 $E_{1} \times \cdots \times E_{n} \times X_{n+1} \times X_{n+2} \times \cdots$ 的子集生成的 (最小) $\sigma-$ 代数, 这里 $E_{m} \subseteq X_{m}=$ $\{0,1\}, m=1, \ldots, n, n=1,2, \ldots$. 则 $X$ 上存在唯一的概率测度 $\mu$ (参见文献 [18]) 满足

(i) $\mathscr{A}$ 恰是全体 $\mu$-可测集之集.

(ii) 对于 $\prod_{m=1}^{n} X_{m}(n \in N)$ 中的任一子集 $E, E \times \prod_{m=n+1}^{\infty} X_{m}$ 都是 $\mu$ - 可测的, 且

$$
\mu\left(E \times \prod_{m=n+1}^{\infty} X_{m}\right)=\left(\mu_{1} \times \cdots \times \mu_{n}\right)(E), \quad n=1,2, \ldots
$$

特别地, 当 $E=E_{1} \times \cdots \times E_{n}$ 时,

$$
\mu\left(E \times \prod_{m=n+1}^{\infty} X_{m}\right)=\left(\mu_{1} \times \cdots \times \mu_{n}\right)(E)=\mu_{1}\left(E_{1}\right) \times \cdots \times \mu_{n}\left(E_{n}\right),
$$

这里 $E_{m} \in \mathscr{P}\left(X_{m}\right), m=1, \ldots, n$. 称 $\mu$ 为 $\mu_{1}, \mu_{2}, \ldots$ 的乘积概率测度, 记为 $\mu=\mu_{1} \times \mu_{2} \times \cdots$. 设 $A \in F(S)$, 定义

$$
\tau_{2}(A)=\mu\left(A^{-1}(1)\right) .
$$

称 $\tau_{2}(A)$ 为 $A$ 的真度, 这里视公式 $A$ 为函数 $A: \Omega \rightarrow\{0,1\}: A(v)=v(A), v \in \Omega$.

设 $A=A\left(p_{1}, \ldots, p_{n}\right) \in F(S)$, 则 $A$ 的赋值 $v(A)$ 只与其中的原子公式 $p_{1}, \ldots, p_{n}$ 的赋值 $v\left(p_{1}\right), \ldots$, $v\left(p_{n}\right)$ 有关 $(v \in \Omega)$, 并且对任二赋值 $u, v \in \Omega$, 只要 $u\left(p_{i}\right)=v\left(p_{i}\right), i=1, \ldots, n$, 则必有 $u(A)=v(A)=$ $\bar{A}\left(v\left(p_{1}\right), \ldots, v\left(p_{n}\right)\right)$. 所以 $A^{-1}(1)$ 必具有形式 $\bar{A}^{-1}(1) \times \prod_{m=n+1}^{\infty} X_{m}$, 即 $A^{-1}(1)=\bar{A}^{-1}(1) \times \prod_{m=n+1}^{\infty} X_{m}$. 从而 (3) 式可简化为

$$
\begin{aligned}
\tau_{2}(A) & =\mu\left(A^{-1}(1)\right)=\mu\left(\bar{A}^{-1}(1) \times \prod_{m=n+1}^{\infty} X_{m}\right) \\
& =\left(\mu_{1} \times \cdots \times \mu_{n}\right)\left(\bar{A}^{-1}(1)\right) \\
& =\left(\mu_{1} \times \cdots \times \mu_{n}\right)\left(\left\{\left(x_{1}, \ldots, x_{n}\right) \mid\left(x_{1}, \ldots, x_{n}\right) \in \bar{A}^{-1}(1)\right\}\right) \\
& =\sum\left\{\left(\mu_{1} \times \cdots \times \mu_{n}\right)\left(\left\{\left(x_{1}, \ldots, x_{n}\right)\right\}\right) \mid\left(x_{1}, \ldots, x_{n}\right) \in \bar{A}^{-1}(1)\right\}
\end{aligned}
$$




$$
\begin{aligned}
& =\sum\left\{\mu_{1}\left(\left\{x_{1}\right\}\right) \times \cdots \times \mu_{n}\left(\left\{x_{n}\right\}\right) \mid\left(x_{1}, \ldots, x_{n}\right) \in \bar{A}^{-1}(1)\right\} \\
& =\sum\left\{\frac{1}{2} \times \cdots \times \frac{1}{2} \mid\left(x_{1}, \ldots, x_{n}\right) \in \bar{A}^{-1}(1)\right\} \\
& =\frac{1}{2^{n}}\left|\bar{A}^{-1}(1)\right| .
\end{aligned}
$$

(4) 式恰是文献 [13] 给出的表达式.

比较 (2) 与 (4) 式, 对具体的公式 $A=A\left(p_{1}, \ldots, p_{n}\right)$ 而言, 当 $(2)$ 式中的概率分布 $P$ 取为均匀概 率分布时, $P(A)=P\left(\bar{A}^{-1}(1)\right)=\sum\left\{P\left(\left\{\left(x_{1}, \ldots, x_{n}\right)\right\}\right) \mid\left(x_{1}, \ldots, x_{n}\right) \in \bar{A}^{-1}(1)\right\}=\frac{1}{2^{n}}\left|\bar{A}^{-1}(1)\right|=\tau_{2}(A)$. 如在例 2.3 (i) 中, 当 $P=(0.25,0.25,0.25,0.25)$ 时, $P(A)=0.75=\tau_{2}(A)$. 所以就局部的具体公式 $A$ 而 言, $\tau_{2}(A)$ 只是 $P(A)$ 在 $P$ 为均匀概率分布时的一个特例. 但值得注意的是, $\tau_{2}$ 是定义在全体公式集 $F(S)$ 上的, 而 $P$ 只是针对具体公式 $A$ 定义的, 因而不能简单地说计量逻辑学是概率逻辑学的特殊情 形, 二者各有优缺点.

最后回忆文献 [15] 定义的公式的随机真度.

定义 $\mathbf{2 . 5}^{[15]}$ 设 $D=\left(P_{1}, P_{2}, \ldots\right)$ 是 $(0,1)$ 中的随机数列, $x=\left(x_{1}, x_{2}, \ldots\right) \in \Omega . \forall i=1,2, \ldots$, 规定

$$
Q_{i}^{x_{i}}= \begin{cases}P_{i}, & x_{i}=1 \\ 1-P_{i}, & x_{i}=0\end{cases}
$$

设 $A=A\left(p_{1}, \ldots, p_{n}\right) \in F(S)$, 定义

$$
\tau_{D}(A)=\sum\left\{Q_{1}^{x_{1}} \times \cdots \times Q_{n}^{x_{n}} \mid\left(x_{1}, \ldots, x_{n}\right) \in \bar{A}^{-1}(1)\right\} .
$$

称 $\tau_{D}(A)$ 为 $A$ 的随机真度.

仅从 (5) 式的定义看不出 $\tau_{D}(A)$ 与 $\tau_{2}(A)$ 的区别与联系, 因为提出 $\tau_{D}(A)$ 的背景不明确, 但它确 实把 $\tau_{2}(A)$ 随机化了. 事实上, 对于随机数列 $D$ 中的任一坐标 $P_{i}, P_{i}$ 可在空间 $X_{i}=\{0,1\}$ 上生成一 个概率测度, 记为 $\mu_{i}$, 满足 $\mu_{i}(\{1\})=P_{i}$. 则 $\mu_{1}, \mu_{2}, \ldots$ 可以在 $\Omega=\{0,1\}^{\omega}$ 上生成唯一的乘积概率测 度 $\mu$ 满足定义 2.4 中的条件 (i) 和 (ii). 所以

$$
\begin{aligned}
\tau_{D}(A) & =\sum\left\{Q_{1}^{x_{1}} \times \cdots \times Q_{n}^{x_{n}} \mid\left(x_{1}, \ldots, x_{n}\right) \in \bar{A}^{-1}(1)\right\} \\
& =\sum\left\{\mu_{1}\left(\left\{x_{1}\right\}\right) \times \cdots \times \mu_{n}\left(\left\{x_{n}\right\}\right) \mid\left(x_{1}, \ldots, x_{n}\right) \in \bar{A}^{-1}(1)\right\} \\
& =\sum\left\{\left(\mu_{1} \times \cdots \times \mu_{n}\right)\left(\left\{\left(x_{1}, \ldots, x_{n}\right)\right\}\right) \mid\left(x_{1}, \ldots, x_{n}\right) \in \bar{A}^{-1}(1)\right\} \\
& =\left(\mu_{1} \times \cdots \times \mu_{n}\right)\left(\bar{A}^{-1}(1)\right)=\mu\left(\bar{A}^{-1}(1) \times \prod_{m=n+1}^{\infty} X_{m}\right) \\
& =\mu\left(A^{-1}(1)\right) .
\end{aligned}
$$

从 (6) 和 (4) 式可以看出, $\tau_{D}(A)$ 和 $\tau_{2}(A)$ 的定义方式是一致的, 都是利用了 $\Omega$ 上的乘积概率测度, 只 不过 (6) 式中的 $\mu$ 未必是均匀的罢了. 特别地当 (6) 式中的 $\mu_{1}, \mu_{2}, \ldots$ 都取为均匀概率测度时, 便有 $\tau_{D}(A)=\tau_{2}(A)$. 但 $(6)$ 式仍要求 $\mu$ 是乘积概率测度, 因而原子公式仍是独立的.

在第 3 节我们将利用 $\Omega$ 上的 Borel 概率测度, 引入公式的概率真度的概念, 把公式的概率 $P(A)$ 、 真度 $\tau_{2}(A)$ 和随机真度 $\tau_{D}(A)$ 均作为特例而纳入到统一框架中. 


\section{3 公式的概率真度及性质}

\section{1 定义及性质}

设 $X_{m}=\{0,1\}$ 是离散拓扑空间 $(m=1,2, \ldots), \Omega=\{0,1\}^{\omega}=\prod_{m=1}^{\infty} X_{m}$ 是通常乘积拓扑空间, 称为赋值空间. 设 $\mathscr{B}(\Omega)$ 和 $\mathscr{B}\left(X_{m}\right)$ 分别是乘积空间 $\Omega$ 和子空间 $X_{m}(m=1,2, \ldots)$ 上的全体 Borel 集 之集, 即由相应空间中的全体拓扑闭集生成的 (最小) $\sigma$ - 代数. 易见 $\mathscr{B}\left(X_{m}\right)=\mathscr{P}\left(X_{m}\right)(m=1,2, \ldots)$. 由文献 [19] 中的引理 8.2.4 知 $\mathscr{B}(\Omega)$ 恰是 $\Omega$ 的拓扑基生成的 $\sigma$ - 代数, 从而 $\mathscr{B}(\Omega)$ 恰是定义 2.4 给出 的 $\mathscr{A}$. 赋值空间 $\Omega$ 上的一个 Borel 概率测度 $\mu$ 是指 $\mu$ 是定义在 $\mathscr{B}(\Omega)$ 上的概率测度, 即, $\mu(\emptyset)=0$, $\mu(\Omega)=1, \mu\left(\bigcup_{k=1}^{\infty} E_{k}\right)=\sum_{k=1}^{\infty} \mu\left(E_{k}\right)$, 这里 $E_{k} \in \mathscr{B}(\Omega)$ 且当 $i \neq j$ 时, $E_{i} \cap E_{j}=\emptyset, i, j, k=1,2, \ldots$ (类 似地定义子空间 $X_{m}=\{0,1\}$ 或有限乘积空间 $\{0,1\}^{n}$ 上的 Borel 概率测度). 则 ( $\left.\Omega, \mathscr{B}(\Omega), \mu\right)$ 是一个 Borel 概率测度空间, 且对任一子集 $E \in \mathscr{B}\left(\{0,1\}^{n}\right)=\mathscr{P}\left(\prod_{m=1}^{n} X_{m}\right), E \times \prod_{m=n+1}^{\infty} X_{m}$ 都是 $\mu$ - 可测 的. 由定义及以上分析知, (3) 和 (6) 式中的概率测度 $\mu$ 都是 Borel 型的.

定义 3.1 设 $A \in F(S), \mu$ 是赋值空间 $\Omega$ 上的 Borel 概率测度. 定义

$$
\tau_{\mu}(A)=\mu\left(A^{-1}(1)\right)
$$

则称 $\tau_{\mu}(A)$ 为 $A$ 的 $\mu$ - 概率真度, 简称 $\mu$ - 真度.

注 3.2 (i) 正像在定义 2.4 中那样, 定义 3.1 仍把每个公式 $A$ 视为函数 $A: \Omega \rightarrow\{0,1\}, A(v)=$ $v(A), v \in \Omega$. 注意, 对任一公式 $A, A: \Omega \rightarrow\{0,1\}$ 是从乘积拓扑空间 $\Omega$ 到离散拓扑空间 $\{0,1\}$ 的连续 函数, 从而是 $\Omega$ 上的 Borel 可测函数. 所以公式 $A$ 也可视为概率空间 $(\Omega, \mathscr{B}(\Omega), \mu)$ 上的一个仅取 0 和 1 两个值的离散型随机变量函数. 又, $\tau_{\mu}(A)=\mu\left(A^{-1}(1)\right)=1 \cdot \mu\left(A^{-1}(1)\right)+0 \cdot \mu\left(A^{-1}(0)\right)=\int_{\Omega} A(v) \mathrm{d} \mu$ 恰是随机变量函数 $A$ 的数学期望. 由于 $A$ 只取 0 和 1 两个值, 且取值为 1 时表示公式 $A$ 为真, 因此 $\tau_{\mu}(A)$ 反映了公式 $A$ 为真的程度, 所以称 $\tau_{\mu}(A)$ 为 $A$ 的 $\mu$ - 真度是合理的.

(ii) 由 (7) 式知两个逻辑等价的公式有相同的 $\mu$ - 真度. 设 $A=A\left(p_{i_{1}}, \ldots, p_{i_{t}}\right)$ 是由原子公式 $p_{i_{1}}, \ldots, p_{i_{t}}$ 构成的公式, 令 $n=\max \left\{i_{j} \mid j=1, \ldots, t\right\}$, 构造公式 $B=B\left(p_{1}, \ldots, p_{n}\right)=A \wedge\left(\bigwedge_{i=1}^{n}\left(p_{i} \vee\right.\right.$ $\left.\neg p_{i}\right)$ ). 则 $B$ 与 $A$ 逻辑等价, 从而 $\tau_{\mu}(A)=\tau_{\mu}(B)$. 在计算公式的 $\mu$ - 真度时, 像 $B$ 这样由前 $n$ 个原子公 式 $p_{1}, \ldots, p_{n}$ 构成的公式有其方便之处. 所以在不影响公式 $A$ 的 $\mu$ - 真度值且又方便计算时, 总假定 $A$ 由 $p_{1}, \ldots, p_{n}$ 构成, 即 $A=A\left(p_{1}, \ldots, p_{n}\right)$. 在前一节我们也是这么做的, 后面仍这么做. 同理, 当只涉 及到有限个公式 $A_{1}, \ldots, A_{m}$ 时, 可取 $n$ 适当大, 并假定 $A_{1}, \ldots, A_{m}$ 都由 $p_{1}, \ldots, p_{n}$ 构成.

(iii) 设 $A=A\left(p_{1}, \ldots, p_{n}\right)$. 则 $A^{-1}(1)=\bar{A}^{-1}(1) \times \prod_{m=n+1}^{\infty} X_{m}$, 从而 $\tau_{\mu}(A)=\mu\left(\bar{A}^{-1}(1) \times\right.$ $\left.\prod_{m=n+1}^{\infty} X_{m}\right)$. 若定义 $\mu(n): \mathscr{P}\left(\{0,1\}^{n}\right) \rightarrow[0,1]:$

$$
\mu(n)(E)=\mu\left(E \times \prod_{m=n+1}^{\infty} X_{m}\right), \quad E \in \mathscr{P}\left(\{0,1\}^{n}\right) .
$$

则 $\mu(n)$ 是有限乘积空间 $\{0,1\}^{n}$ 上的 Borel 概率测度, 称为 $\mu$ 在 $\{0,1\}^{n}$ 上的限制. 此时 (7) 式可简 化为

$$
\tau_{\mu}(A)=\mu\left(A^{-1}(1)\right)=\mu\left(\bar{A}^{-1}(1) \times \prod_{m=n+1}^{\infty} X_{m}\right)=\mu(n)\left(\bar{A}^{-1}(1)\right) .
$$

上面的转化说明赋值空间 $\Omega$ 上的任一 Borel 概率测度 $\mu$ 都可诱导出有限乘积空间 $\{0,1\}^{n}$ 上的 一个 Borel 概率测度 $\mu(n)$. 反过来, 设 $P$ 是 $\{0,1\}^{n}$ 上的任一 Borel 概率测度. 令 $\mu_{m}$ 是 $X_{m}=\{0,1\}$ 
上的均匀概率测度 (事实上, 只要求 $\mu_{m}$ 是 $X_{m}$ 上的 Borel 概率测度即可) $(m=n+1, n+2, \ldots)$. 由 于 $\Omega$ 也可视为 $\{0,1\}^{n}, X_{n+1}, X_{n+2}, \ldots$ 的无穷乘积空间, 所以 $P, \mu_{n+1}, \mu_{n+2}, \ldots$ 也可以在 $\Omega$ 上生成唯 一的 Borel 乘积概率测度 $\mu$, 使得对任一子集 $E \subseteq\{0,1\}^{k}(k \geqslant n), E \times \prod_{m=k+1}^{\infty} X_{m}$ 都是 $\mu$ - 可测的且 $\mu\left(E \times \prod_{m=k+1}^{\infty} X_{m}\right)=\left(P \times \mu_{n+1} \times \cdots \times \mu_{k}\right)(E)$. 特别地, 若 $k=n$, 则 $P(E)=\mu\left(E \times \prod_{m=n+1}^{\infty} X_{m}\right)=$ $\mu(n)(E)$. 这说明 $P$ 恰好等于按上述方式生成的 $\Omega$ 上的 Borel 概率测度 $\mu$ 在 $\{0,1\}^{n}$ 上的限制 $\mu(n)$. 正是由于这种限制才让我们将清楚地看到 $\tau_{\mu}(A)$ 与 (1) 式中的 $P(A)$ 间的区别和联系, 并将 $P(A)$ 纳 入到统一的框架中, 从而弥补概率逻辑学缺乏整体性的不足. 今后, 我们总用 $\mu(n)$ 表示有限乘积空间 $\{0,1\}^{n}$ 上的任一 Borel 概率测度且设 $\mu(n)$ 是 $\Omega$ 上的 Borel 概率测度 $\mu$ 在 $\{0,1\}^{n}$ 上的限制.

(iv) 设 $A=A\left(p_{1}, \ldots, p_{n}\right) \in F(S), \mu(n)$ 是 (状态描述集) $\{0,1\}^{n}$ 上的概率测度, 则 $\mu(n)$ 自然是 $\{0,1\}^{n}$ 上的概率分布, 所以 $\tau_{\mu}(A)=\mu(n)\left(\bar{A}^{-1}(1)\right)=\sum\left\{\mu(n)\left(\left\{\left(x_{1}, \ldots, x_{n}\right)\right\}\right) \mid\left(x_{1}, \ldots, x_{n}\right) \in \bar{A}^{-1}(1)\right\}$. 由 (2) 式知 $\tau_{\mu}(A)$ 正是概率逻辑学意义下的 $A$ 的概率. 与 (2) 式不同的是, 这里的 $\tau_{\mu}$ 是定义在全体 公式集 $F(S)$ 上的, 从而是具有整体性的. 又由 (iii) 知当 $\mu$ 取遍 $\Omega$ 上的 Borel 概率测度时 $\mu(n)$ 也可 以取遍 $\{0,1\}^{n}$ 上的概率分布, 所以 $(2)$ 式中 $A$ 的概率 $P(A)$ 总可用 $\tau_{\mu}(A)$ 表示.

(v) 设 $A=A\left(p_{1}, \ldots, p_{n}\right) \in F(S), \mu$ 是 $\Omega$ 的每个子空间 $X_{m}$ 上的 Borel 概率测度 $\mu_{m}(m=1,2, \ldots)$ 生成的 Borel 乘积概率测度, 即 $\mu=\mu_{1} \times \mu_{2} \times \cdots$, 则

$$
\begin{aligned}
\tau_{\mu}(A) & =\mu(n)\left(\bar{A}^{-1}(1)\right)=\left(\mu_{1} \times \cdots \times \mu_{n}\right)\left(\bar{A}^{-1}(1)\right) \\
& =\sum\left\{\left(\mu_{1} \times \cdots \times \mu_{n}\right)\left(\left\{\left(x_{1}, \ldots, x_{n}\right)\right\}\right) \mid\left(x_{1}, \ldots, x_{n}\right) \in \bar{A}^{-1}(1)\right\} \\
& =\sum\left\{\mu_{1}\left(\left\{x_{1}\right\}\right) \times \cdots \times \mu_{n}\left(\left\{x_{n}\right\}\right) \mid\left(x_{1}, \ldots, x_{n}\right) \in \bar{A}^{-1}(1)\right\} .
\end{aligned}
$$

(9) 式恰是 (6) 式. 可见文献 [15] 定义的随机真度只是本文定义的 $\mu$ - 真度在 $\mu$ 为乘积概率测度时的 特例. 特别地, 当每个 $\mu_{m}$ 都是均匀概率测度时, 由 (9) 式知 $\tau_{\mu}(A)=\frac{1}{2^{n}}\left|\bar{A}^{-1}(1)\right|=\tau_{2}(A)$, 即得 (4) 式.

(vi) 设 $v=\left(v_{1}, v_{2}, \ldots\right) \in \Omega$. 令 $\mu_{m}(\emptyset)=0, \mu_{m}\left(X_{m}\right)=1, \mu_{m}(\{1\})=v_{m}, \mu_{m}(\{0\})=1-v_{m}(m=$ $1,2, \ldots)$, 且 $\mu=\mu_{1} \times \mu_{2} \times \cdots$, 则 $\tau_{\mu}=v$. 这说明每个赋值 $v \in \Omega$ 是定义 3.1 意义下的概率真度函数.

从注 3.2 (iii) 至 (vi) 可以看出, 本文讨论的公式的 $\mu$ - 真度理论比概率逻辑学研究的公式的概率 理论和计量逻辑学研究的真度理论都要宽泛的多.

例 3.3 设 $A=p_{1}, B=p_{2} \rightarrow p_{3}, C=p_{1} \vee p_{2} \vee p_{3}, D=p_{1} \wedge p_{2} \wedge p_{3}$. 求 $\tau_{\mu}(A), \tau_{\mu}(B), \tau_{\mu}(C)$ 和 $\tau_{\mu}(D)$.

解 因为公式 $A, B, C, D$ 只涉及到 3 个原子公式 $p_{1}, p_{2}, p_{3}$, 由 (8) 式知它们的 $\mu$ - 真度只和 $\{0,1\}^{3}$ 上的 Borel 概率测度 $\mu(3)$ 有关. 所以我们不必关心整个 $\mu$, 只考虑 $\mu(3)$ 即可.

(i) 设 $\mu(3)(\{(1,1,1)\})=\mu(3)(\{(1,1,0)\})=0.2, \mu(3)\left(\left\{\left(x_{1}, x_{2}, x_{3}\right)\right\}\right)=0.1,\left(x_{1}, x_{2}, x_{3}\right) \in\{0,1\}^{3}$ 且 $\left(x_{1}, x_{2}, x_{3}\right) \neq(1,1,1),\left(x_{1}, x_{2}, x_{3}\right) \neq(1,1,0)$, 则

$\tau_{\mu}(A)=\mu(3)\left(\bar{A}^{-1}(1)\right)=\mu(3)(\{(1,1,1),(1,1,0),(1,0,1),(1,0,0)\})=0.6 ;$

$\tau_{\mu}(B)=\mu(3)\left(\bar{B}^{-1}(1)\right)=\mu(3)(\{(1,1,1),(1,0,1),(1,0,0),(0,1,1),(0,0,1),(0,0,0)\}=0.7 ;$

$\tau_{\mu}(C)=\mu(3)\left(\bar{C}^{-1}(1)\right)=\mu(3)\left(\{0,1\}^{3}-\{(0,0,0)\}\right)=0.9 ;$

$\tau_{\mu}(D)=\mu(3)(\{(1,1,1)\})=0.2$.

(ii) 设 $\mu(3)$ 是 $\{0,1\}^{3}$ 上的均匀概率分布, 则 $\tau_{\mu}(A)=0.5, \tau_{\mu}(B)=0.75, \tau_{\mu}(C)=0.875, \tau_{\mu}(D)=$ 0.125 , 这与文献 $[13,14]$ 中的相应结果一致.

命题 3.4 设 $\mu$ 是 $\Omega$ 上的 Borel 概率测度, 则

(i) $\forall A \in F(S)$, 若 $A$ 是重言式 (矛盾式), 则 $\tau_{\mu}(A)=1\left(\tau_{\mu}(A)=0\right.$ ). 
(ii) 设 $A=A\left(p_{1}, \ldots, p_{n}\right) \in F(S)$, 并假定 $\mu$ 满足 $\forall\left(x_{1}, \ldots, x_{n}\right) \in\{0,1\}^{n}, \mu(n)\left(\left\{\left(x_{1}, \ldots, x_{n}\right)\right\}\right) \neq 0$. 若 $\tau_{\mu}(A)=1\left(\tau_{\mu}(A)=0\right)$, 则 $A$ 是重言式 (矛盾式).

(iii) 设 $\mu$ 满足 $\forall n \in N, \forall\left(x_{1}, \ldots, x_{n}\right) \in\{0,1\}^{n}, \mu(n)\left(\left\{\left(x_{1}, \ldots, x_{n}\right)\right\}\right) \neq 0 . \forall B \in F(S)$, 若 $\tau_{\mu}(B)=$ $1\left(\tau_{\mu}(B)=0\right)$, 则 $B$ 是重言式 (矛盾式).

证明 (i) 不妨设 $A=A\left(p_{1}, \ldots, p_{n}\right)$, 由 $A$ 是重言式知 $\bar{A}^{-1}(1)=\{0,1\}^{n}$, 所以 $\tau_{\mu}(A)=\mu(n)$ $\left(\bar{A}^{-1}(1)\right)=1$. 类似可证若 $A$ 是矛盾式, 则 $\tau_{\mu}(A)=0$.

(ii) 设 $A=A\left(p_{1}, \ldots, p_{n}\right)$ 且 $\tau_{\mu}(A)=1$, 即 $\mu(n)\left(\bar{A}^{-1}(1)\right)=1$. 为证 $A$ 是重言式, 只须证 $\bar{A}^{-1}(1)=$ $\{0,1\}^{n}$. 假设 $\bar{A}^{-1}(1) \neq\{0,1\}^{n}$, 则存在 $\left(x_{1}, \ldots, x_{n}\right) \in\{0,1\}^{n}$ 使得 $\left(x_{1}, \ldots, x_{n}\right) \notin \bar{A}^{-1}(1)$. 从而 $\tau_{\mu}(A)=\mu(n)\left(\bar{A}^{-1}(1)\right) \leqslant \mu(n)\left(\{0,1\}^{n}-\left\{\left(x_{1}, \ldots, x_{n}\right)\right\}\right)=1-\mu(n)\left(\left\{\left(x_{1}, \ldots, x_{n}\right)\right\}\right)<1$, 矛盾! 所以 $\bar{A}^{-1}(1)=\{0,1\}^{n}$, 从而 $A$ 是重言式. 类似可证 $\tau_{\mu}(A)=0$ 时的情形.

(iii) 是 (ii) 的直接推论.

在下面的命题中我们罗列 $\tau_{\mu}$ 的基本性质. 为便于集中比较, 把命题 3.4 中的 (i) 也列了出来.

命题 3.5 设 $A, B \in F(S), \mu$ 是 $\Omega$ 上的 Borel 概率测度, 则

(i) $0 \leqslant \tau_{\mu}(A) \leqslant 1$.

(ii) 若 $A$ 是重言式 (矛盾式), 则 $\tau_{\mu}(A)=1\left(\tau_{\mu}(A)=0\right)$.

(iii) 若 $A$ 与 $B$ 逻辑等价, 则 $\tau_{\mu}(A)=\tau_{\mu}(B)$.

(iv) $\tau_{\mu}(\neg A)=1-\tau_{\mu}(A)$.

(v) $\tau_{\mu}(A)+\tau_{\mu}(B)=\tau_{\mu}(A \vee B)+\tau_{\mu}(A \wedge B)$.

(vi) $1+\tau_{\mu}(A \wedge B)=\tau_{\mu}(A)+\tau_{\mu}(A \rightarrow B)$.

(vii) $\tau_{\mu}(A)+\tau_{\mu}(A \rightarrow B)=\tau_{\mu}(B)+\tau_{\mu}(B \rightarrow A)$.

(viii) 若 $=A \rightarrow B$, 则 $\tau_{\mu}(A) \leqslant \tau_{\mu}(B)$.

(ix) $\tau_{\mu}(B) \leqslant \tau_{\mu}(A \rightarrow B)$.

(x) $\tau_{\mu}(A \wedge B) \leqslant \min \left\{\tau_{\mu}(A), \tau_{\mu}(B)\right\} \leqslant \max \left\{\tau_{\mu}(A), \tau_{\mu}(B)\right\} \leqslant \tau_{\mu}(A \vee B)$.

证明 以 (v) 为例进行证明, 其他各条可类似验证. $(A \vee B)^{-1}(1)=\{v \in \Omega \mid v(A \vee B)=1\}=$ $\{v \in \Omega \mid v(A)=1$ 或 $v(B)=1\}=\{v \in \Omega \mid v(A)=1\} \cup\{v \in \Omega \mid v(B)=1\}=A^{-1}(1) \cup B^{-1}(1)$. 类似 地, $(A \wedge B)^{-1}(1)=A^{-1}(1) \cap B^{-1}(1)$. 从而

$$
\begin{aligned}
\tau_{\mu}(A \vee B) & =\mu\left((A \vee B)^{-1}(1)\right)=\mu\left(A^{-1}(1) \cup B^{-1}(1)\right) \\
& =\mu\left(A^{-1}(1)\right)+\mu\left(B^{-1}(1)\right)-\mu\left(A^{-1}(1) \cap B^{-1}(1)\right) \\
& =\mu\left(A^{-1}(1)\right)+\mu\left(B^{-1}(1)\right)-\mu\left((A \wedge B)^{-1}(1)\right) \\
& =\tau_{\mu}(A)+\tau_{\mu}(B)-\tau_{\mu}(A \wedge B) .
\end{aligned}
$$

注意, 在证明 $\tau_{\mu}$ 的一般性质时, 使用其原始定义 (7) 式是比较方便的, 但当计算具体公式的 $\mu$ - 真 度时自然是使用 (8) 式更方便些, 见例 3.3 .

命题 3.6 设 $A, B, C \in F(S), \mu$ 是 $\Omega$ 上的 Borel 概率测度, $\alpha, \beta \in[0,1]$, 则

(i) 若 $\tau_{\mu}(A) \geqslant \alpha, \tau_{\mu}(A \rightarrow B) \geqslant \beta$, 则 $\tau_{\mu}(B) \geqslant \alpha+\beta-1$.

(ii) 若 $\tau_{\mu}(A \rightarrow B) \geqslant \alpha, \tau_{\mu}(B \rightarrow C) \geqslant \beta$, 则 $\tau_{\mu}(A \rightarrow C) \geqslant \alpha+\beta-1$.

分别把规则 (i) 和 (ii) 称为关于 $\tau_{\mu}$ 的 MP 规则和 HS 规则.

证明 (i) 由命题 3.5 (vii) 知

$$
\tau_{\mu}(B)=\tau_{\mu}(A)+\tau_{\mu}(A \rightarrow B)-\tau_{\mu}(B \rightarrow A) \geqslant \alpha+\beta-\tau_{\mu}(B \rightarrow A) \geqslant \alpha+\beta-1 .
$$


(ii) 由 $(A \rightarrow B) \rightarrow((B \rightarrow C) \rightarrow(A \rightarrow C))$ 是重言式, 所以由命题 3.5(viii) 知 $\tau_{\mu}((B \rightarrow C) \rightarrow(A \rightarrow$ $C)) \geqslant \tau_{\mu}(A \rightarrow B) \geqslant \alpha$. 用 (i) 得

$$
\tau_{\mu}(A \rightarrow C) \geqslant \tau_{\mu}(B \rightarrow C)+\tau_{\mu}((B \rightarrow C) \rightarrow(A \rightarrow C))-1 \geqslant \beta+\alpha-1=\alpha+\beta-1 .
$$

推论 3.7 设 $A, B \in F(S), \mu$ 是 $\Omega$ 上的 Borel 概率测度, 则

(i) 若 $\tau_{\mu}(A \rightarrow B)=1$, 则 $\tau_{\mu}(A) \leqslant \tau_{\mu}(B)$.

(ii) 若 $\tau_{\mu}(A)=1, \tau_{\mu}(A \rightarrow B)=1$, 则 $\tau_{\mu}(B)=1$.

(iii) 若 $\tau_{\mu}(A \rightarrow B)=1, \tau_{\mu}(B \rightarrow C)=1$, 则 $\tau_{\mu}(A \rightarrow C)=1$.

\section{2 逻辑闭理论与拓扑闭集}

定义 3.8 设 $\mu$ 是 $\Omega$ 上的 Borel 概率测度, 定义

$$
\operatorname{ker}\left(\tau_{\mu}\right)=\left\{A \in F(S) \mid \tau_{\mu}(A)=1\right\}
$$

称 $\operatorname{ker}\left(\tau_{\mu}\right)$ 为 $\tau_{\mu}$ 的核.

定理 $3.9 \operatorname{ker}\left(\tau_{\mu}\right)$ 是二值命题逻辑中的相容逻辑闭理论, 即

$$
\operatorname{ker}\left(\tau_{\mu}\right)=D\left(\operatorname{ker}\left(\tau_{\mu}\right)\right) \text {, 且 } \perp \notin \operatorname{ker}\left(\tau_{\mu}\right) .
$$

证明 (i) 设 $A \in F(S)$. 若 $A$ 是公理, 则显然有 $A \in \operatorname{ker}\left(\tau_{\mu}\right)$.

(ii) 设 $A, A \rightarrow B \in \operatorname{ker}\left(\tau_{\mu}\right)$, 则 $\tau_{\mu}(A)=1, \tau_{\mu}(A \rightarrow B)=1$. 由推论 $3.7($ ii $)$ 知 $\tau_{\mu}(B)=1$, 从而 $B \in \operatorname{ker}\left(\tau_{\mu}\right)$.

由 (i) 和 (ii) 便得 $\operatorname{ker}\left(\tau_{\mu}\right)=D\left(\operatorname{ker}\left(\tau_{\mu}\right)\right)$, 即, $\operatorname{ker}\left(\tau_{\mu}\right)$ 是逻辑闭的. 又显然矛盾式 $\perp \notin \operatorname{ker}\left(\tau_{\mu}\right)$, 所以 $\operatorname{ker}\left(\tau_{\mu}\right)$ 是相容的.

下面讨论定理 3.9 的逆问题, 即, 二值命题逻辑中的相容的逻辑闭理论是否都是某 $\tau_{\mu}$ 的核 $\operatorname{ker}\left(\tau_{\mu}\right)$ ? 为回答上述问题, 我们先研究逻辑理论的模型的性质.

定义 3.10 设 $\Sigma \subseteq \Omega=\{0,1\}^{\omega}$. 若 $\Sigma$ 满足 $\forall u=\left(u_{1}, u_{2}, \ldots\right) \in \Omega-\Sigma$, 存在 $n \in N$ 使得 $\forall v=\left(v_{1}, v_{2}, \ldots\right) \in \Sigma,\left(u_{1}, \ldots, u_{n}\right) \neq\left(v_{1}, \ldots, v_{n}\right)$, 则称 $\Sigma$ 具有有限截断分离性质, 简称有限分离性质.

例如, $\Sigma=\emptyset, \Sigma=\{(1,1, \ldots)\}$ 和 $\Sigma=\Omega$ 都具有有限分离性质, 然而 $\Sigma=\Omega-\{(1,1, \ldots)\}$ 不具有 此性质. 我们将通过具有有限分离性质的 $\Omega$ 的子集建立逻辑闭理论和 $\Omega$ 中的拓扑闭集间的一一对应 关系, 并最终证明二值命题逻辑中的相容逻辑闭理论都是某 $\tau_{\mu}$ 的核 $\operatorname{ker}\left(\tau_{\mu}\right)$.

定理 3.11 设 $\Gamma$ 是一理论, $\Sigma$ 是 $\Gamma$ 的模型之集, 即 $\Sigma=\{v \in \Omega \mid \forall A \in \Gamma, v(A)=1\}$, 则 $\Sigma$ 具有 有限分离性质.

证明 用反证法. 假设 $\Sigma$ 不具有该性质, 则存在 $u=\left(u_{1}, u_{2}, \ldots\right) \in \Omega-\Sigma$ 使得 $\forall n \in N$, 存在 $v=\left(v_{1}, v_{2}, \ldots\right) \in \Sigma,\left(u_{1}, \ldots, u_{n}\right)=\left(v_{1}, \ldots, v_{n}\right)$. 任取 $A \in \Gamma$, 不妨设 $A=A\left(p_{1}, \ldots, p_{n}\right)$, 则

$$
1=v(A)=\bar{A}\left(v\left(p_{1}\right), \ldots, v\left(p_{n}\right)\right)=\bar{A}\left(u\left(p_{1}\right), \ldots, u\left(p_{n}\right)\right)=u(A) .
$$

由 $A$ 的任意性知, $u \in \Sigma$, 矛盾!

定理 3.12 设 $\Sigma \subseteq \Omega$ 具有有限分离性质, 则存在理论 $\Gamma$ 使得 $\Sigma$ 恰是 $\Gamma$ 的模型之集. 
证明 若 $\Sigma=\emptyset$, 取 $\Gamma=\left\{p_{1}, \neg p_{1}\right\}$ 即可. 以下设 $\Sigma \neq \emptyset . \forall n \in N$, 令 $\Sigma(n)=\left\{\left(v_{1}, \ldots, v_{n}\right) \mid v=\right.$ $\left.\left(v_{1}, v_{2}, \ldots\right) \in \Sigma\right\}$. 规定 $p^{1}=p, p^{0}=\neg p, p \in S$. 定义

$$
\Gamma=\left\{\vee\left\{p_{1}^{v_{1}} \wedge \cdots \wedge p_{n}^{v_{n}} \mid\left(v_{1}, \ldots, v_{n}\right) \in \Sigma(n)\right\} \mid n \in N\right\} .
$$

注意, 由于 $\forall n \in N, \Sigma(n)$ 是有限集, 所以 $\Gamma$ 中的每个公式都是定义好的, 从而 $\Gamma$ 是一个理论. 易验证 $\Sigma$ 恰是 $\Gamma$ 的模型之集.

定理 3.11 说明二值命题逻辑中每个逻辑理论的模型之集都具有有限分离性质, 从而逻辑闭理论 的模型之集当然也具有有限分离性质. 定理 3.12 对于给定的具有有限分离性质的 $\Sigma$ 构造出了理论 $\Gamma$, 其模型之集恰为 $\Sigma$. 值得指出的是, 模型之集为 $\Sigma$ 的理论是非常多的, 比如满足 $\Gamma \subseteq \Gamma^{\prime} \subseteq D(\Gamma)$ 的理 论 $\Gamma^{\prime}$ 的模型之集都是 $\Sigma$. 但模型之集为 $\Sigma$ 的逻辑闭理论只有一个, 即为 $D(\Gamma)$, 这是因为

命题 3.13 设 $\Gamma_{1}, \Gamma_{2}$ 是两个不同的理论, $\Sigma_{1}, \Sigma_{2}$ 分别是它们的模型之集, 则

$$
D\left(\Gamma_{1}\right)=D\left(\Gamma_{2}\right) \text { iff } \Sigma_{1}=\Sigma_{2} .
$$

证明 由二值命题逻辑的强完备性定理知这是显然的.

推论 3.14 逻辑闭理论和 $\Omega$ 中具有有限分离性质的子集是一一对应的.

下面证明 $\Omega$ 中具有有限分离性质的子集与 $\Omega$ 作为乘积拓扑空间中的拓扑闭集也是一一对应的. 注意 $\Omega$ 作为乘积拓扑空间是可度量化的, 其度量 $\rho^{[20]}$ 为

$$
\rho(u, v)=\max \left\{\frac{\left|u_{i}-v_{i}\right|}{i} \mid i=1,2, \ldots\right\},
$$

$u=\left(u_{1}, u_{2}, \ldots\right), v=\left(v_{1}, v_{2}, \ldots\right) \in \Omega$.

定理 3.15 设 $\Sigma \subseteq \Omega$, 则 $\Sigma$ 具有有限分离性质 iff $\Sigma$ 是 $(\Omega, \rho)$ 中的闭集.

证明 设 $\Sigma$ 具有有限分离性质. 为证明 $\Sigma$ 是拓扑闭的, 只需证 $\forall u \in \Omega-\Sigma$, 存在 $\varepsilon>0$ 使得 $\rho(u, \Sigma)>\varepsilon$. 任取 $u \in \Omega-\Sigma$, 则存在 $n \in N$ 使得 $\forall v=\left(v_{1}, v_{2}, \ldots\right) \in \Sigma,\left(u_{1}, \ldots, u_{n}\right) \neq\left(v_{1}, \ldots, v_{n}\right)$. 取 $\varepsilon=\frac{1}{n+1}$, 则 $\forall v=\left(v_{1}, v_{2}, \ldots\right), \rho(u, v) \geqslant \frac{1}{n}>\frac{1}{n+1}=\varepsilon$, 从而 $\rho(u, \Sigma) \geqslant \frac{1}{n}>\varepsilon$. 所以 $\Sigma$ 是闭的.

反过来, 设 $\Sigma$ 是闭的. 则 $\forall u \in \Omega-\Sigma$, 存在 $\varepsilon(0<\varepsilon<1)$ 使得 $\rho(u, \Sigma)>\varepsilon$. 取 $n=\left[\frac{1}{\varepsilon}\right]+1$, 则 $\varepsilon>\frac{1}{n}$. 所以 $\forall v \in \Sigma, \rho(u, v)>\frac{1}{n}$, 从而 $\left(u_{1}, \ldots, u_{n}\right) \neq\left(v_{1}, \ldots, v_{n}\right)$. 这便证得 $\Sigma$ 具有有限分离性质.

由推论 3.14 和定理 3.15 知

定理 3.16 二值命题逻辑中的逻辑闭理论和 $\Omega$ 中的拓扑闭集是一一对应的.

由定理 3.16 知 $\Omega$ 中最小的闭集 $\emptyset$ 与最大的 (不相容) 逻辑闭理论 $F(S)$ 相对应, 最大的拓扑闭集 $\Omega$ 与最小相容逻辑闭理论 $D(\emptyset)$ 相对应. 自然地, $\Omega$ 中极小闭集 (即单点集) 必与极大相容理论 (极大 相容理论一定是逻辑闭的 ${ }^{[21]}$ ) 相对应. 由此一一对应可将空间 $\Omega$ 上的度量 $\rho$ 转移到全体极大相容理 论之集上从而给出其拓扑刻画. 再由 (10) 式就可给出每个极大相容理论的结构刻画.

定理 $3.17 \forall v=\left(v_{1}, v_{2}, \ldots\right) \in \Omega$, 定义 $\Gamma_{v}=D\left(\left\{p_{1}^{v_{1}}, p_{1}^{v_{1}} \wedge p_{2}^{v_{2}}, \ldots\right\}\right)$, 则

(i) $M=\left\{\Gamma_{v} \mid v \in \Omega\right\}$ 是二值命题逻辑中全体极大相容理论之集.

(ii) 在 $M$ 上规定 $d\left(\Gamma_{u}, \Gamma_{v}\right)=\rho(u, v), u, v \in \Omega$, 这里 $\rho$ 由 (11) 式定义, 则 $(M, d)$ 是一 Cantor 空间 ${ }^{222]}$.

请读者自行验证, $\forall v=\left(v_{1}, v_{2}, \ldots\right) \in \Omega$, 定理 3.17 中的极大相容理论 $\Gamma_{v}$ 可简化为 $\Gamma_{v}=D\left(\left\{p_{1}^{v_{1}}, p_{2}^{v_{2}}\right.\right.$, ...\}), 这便与文献 $[23]$ 给出的结构相一致.

下面我们证明 
定理 3.18 设 $\Gamma$ 是二值命题逻辑中的相容逻辑闭理论, 则存在 $\Omega$ 上的 Borel 概率测度 $\mu$ 使得

$$
\Gamma=\operatorname{ker}\left(\tau_{\mu}\right)
$$

证明 设 $\Gamma$ 是相容逻辑闭理论, 令 $\Sigma$ 是 $\Gamma$ 的全体模型之集. 则由定理 3.11 及 3.15 知 $\Sigma$ 是 $\Omega$ 中 的非空拓扑闭集. 视 $\Sigma$ 为 $\Omega$ 的拓扑子空间, 则 $\mathscr{B}(\Sigma)=\mathscr{B}(\Omega) \cap \Sigma=\{\Delta \cap \Sigma \mid \Delta \in \mathscr{B}(\Omega)\}$. 任取 $\Sigma$ 上 的一 Borel 概率测度 $\mu_{0}$ 满足 $\mu_{0}(\Lambda)=1$ iff $\Lambda=\Sigma$, 这里 $\Lambda$ 为 $\Sigma$ 中的拓扑闭集. 定义 $\mu: \mathscr{B}(\Omega) \rightarrow[0,1]$ :

$$
\mu(\Delta)=\mu_{0}(\Delta \cap \Sigma), \quad \Delta \in \mathscr{B}(\Omega) .
$$

则 $\mu$ 是 $\Omega$ 上的 Borel 概率测度. 任取 $A \in \Gamma$, 则显然 $\Sigma \subseteq A^{-1}(1)$, 所以 $\tau_{\mu}(A)=\mu\left(A^{-1}(1)\right)=\mu(\Sigma)=1$, 从而 $\Gamma \subseteq \operatorname{ker}\left(\tau_{\mu}\right)$. 反过来, 由于 $\Gamma$ 和 $\operatorname{ker}\left(\tau_{\mu}\right)$ 都是逻辑闭的, 为证 $\operatorname{ker}\left(\tau_{\mu}\right) \subseteq \Gamma$, 只须证 $\Gamma$ 的每个模型 $v \in \Sigma$ 都是 $\operatorname{ker}\left(\tau_{\mu}\right)$ 的模型, 即证 $\forall B \in \operatorname{ker}\left(\tau_{\mu}\right), \Sigma \subseteq B^{-1}(1)$. 假设存在公式 $B \in \operatorname{ker}\left(\tau_{\mu}\right), \Sigma \nsubseteq B^{-1}(1)$, 则 $B^{-1}(1) \cap \Sigma$ 是比 $\Sigma$ 真小的闭集. 从而 $\tau_{\mu}(B)=\mu\left(B^{-1}(1)\right)=\mu_{0}\left(B^{-1}(1) \cap \Sigma\right) \neq 1$, 矛盾!

定理 3.9 证明了每个 $\operatorname{ker}\left(\tau_{\mu}\right)$ 都是相容的逻辑闭理论, 而定理 3.18 说明每个相容的逻辑闭理论都 是某 $\tau_{\mu}$ 的核 $\operatorname{ker}\left(\tau_{\mu}\right)$, 从而相容的逻辑闭理论和概率真度函数的核是一一对应的. 注意, 这里并未说相 容的逻辑闭理论和概率真度函数是一一对应的. 事实上, 具有相同核的概率真度函数有很多. 下面给 出具有相同核的概率真度函数的刻画.

定理 3.19 设 $\mu, \nu$ 是 $\Omega$ 上的 Borel 概率测度, 则 $\operatorname{ker}\left(\tau_{\mu}\right)=\operatorname{ker}\left(\tau_{\nu}\right)$ iff $\mu(\Sigma)=\nu(\Sigma)=1$ 且对拓 扑闭集 $\Delta$, 若 $\Delta \subset \Sigma$, 则 $\mu(\Delta)<1, \nu(\Delta)<1$, 这里 $\Sigma$ 是 $\operatorname{ker}\left(\tau_{\mu}\right)$ 的模型之集. 特别地, 当 $\operatorname{ker}\left(\tau_{\mu}\right)$ 为极 大相容理论时,

$$
\operatorname{ker}\left(\tau_{\mu}\right)=\operatorname{ker}\left(\tau_{\nu}\right) \text { iff } \tau_{\mu}=\tau_{\nu} .
$$

证明 由定理 3.18 的证明知, 前半部分是显然的. 当 $\operatorname{ker}\left(\tau_{\mu}\right)$ 为极大相容理论时, 由定理 3.17 知 存在 $u \in \Omega$, 使得 $\Sigma=\{u\}$, 从而 $\mu(\{u\})=\nu(\{u\})=1$. 所以 $\tau_{\mu}=u=\tau_{\nu}$.

但当 $\tau_{\mu}=\tau_{\nu}$ 时有 $\mu=\nu$, 即

定理 3.20 设 $\mu, \nu$ 是 $\Omega$ 上的两个 Borel 概率测度, 则

$$
\tau_{\mu}=\tau_{\nu} \text { iff } \mu=\nu
$$

为证明定理 3.20 , 需要一个引理.

引理 3.21 ${ }^{[18]}$ 设 $\mu, \nu$ 是 $\Omega$ 上的两个 Borel 概率测度, 若对任一拓扑闭集 $\Sigma \subseteq \Omega, \mu(\Sigma)=\nu(\Sigma)$, 则 $\mu=\nu$.

下面证明定理 3.20. 设 $\tau_{\mu}=\tau_{\nu}$. 由引理 3.21 只需证对任一闭集 $\Sigma \subseteq \Omega, \mu(\Sigma)=\nu(\Sigma)$. 假设存 在闭集 $\Sigma_{0} \subseteq \Omega$ 使得 $\mu\left(\Sigma_{0}\right) \neq \nu\left(\Sigma_{0}\right)$. 则存在 $n_{0} \in N$ 使得 $\mu\left(\Sigma_{0}\left(n_{0}\right) \times \prod_{m=n_{0}+1}^{\infty} X_{m}\right) \neq \nu\left(\Sigma_{0}\left(n_{0}\right) \times\right.$ $\left.\prod_{m=n_{0}+1}^{\infty} X_{m}\right)$. 由 (10) 式构造公式 $A_{0}=\vee\left\{p_{1}^{v_{1}} \wedge \cdots \wedge p_{n_{0}}^{v_{n_{0}}} \mid\left(v_{1}, \ldots, v_{n_{0}}\right) \in \Sigma_{0}\left(n_{0}\right)\right\}$. 显然有 $\bar{A}_{0}^{-1}(1)=$ $\Sigma_{0}\left(n_{0}\right)$. 所以 $\tau_{\mu}\left(A_{0}\right)=\mu\left(\Sigma_{0}\left(n_{0}\right) \times \prod_{m=n_{0}+1}^{\infty} X_{m}\right) \neq \nu\left(\Sigma_{0}\left(n_{0}\right) \times \prod_{m=n_{0}+1}^{\infty} X_{m}\right)=\tau_{\nu}\left(A_{0}\right)$, 矛盾! 这便证 得 $\mu=\nu$. 反过来, 若 $\mu=\nu$, 则显然有 $\tau_{\mu}=\tau_{\nu}$. 所以定理 3.20 成立.

定理 3.20 告诉我们, 在二值命题逻辑中按 (7) 式定义的概率真度函数 $\tau_{\mu}$ 与 $\Omega$ 的 Borel 概率测度 $\mu$ 是一样多的. 在第 4 节我们将利用概率学中的 Kolmogorov 公理给出概率真度函数的公理化定义, 并证明公式集上满足 Kolmogorov 公理的任一 $[0,1]$ 值函数都可由赋值空间上的 Borel 概率测度按 (7) 式导出, 从而在二值命题逻辑中只有形如 (7) 式的概率真度函数. 


\section{4 概率真度函数的公理化定义及其表示定理}

本节我们给出 $F(S)$ 上的概率真度函数的公理化定义, 研究其性质及等价刻画条件, 最后证明任 一概率真度函数都由 $\Omega$ 上的 Borel 概率测度按 (7) 式表出.

定义 4.1 (Kolmogorov 公理) 设 $A, B \in F(S), \tau: F(S) \rightarrow[0,1]$ 是一映射且满足:

(K1) $0 \leqslant \tau(A) \leqslant 1$.

(K2) 若 $A$ 是公理, 则 $\tau(A)=1$.

(K3) 若 $A \rightarrow B$ 是定理, 则 $\tau(A) \leqslant \tau(B)$.

(K4) 若 $\{A, B\}$ 作为理论不相容 (简称 $A$ 与 $B$ 不相容), 则 $\tau(A \vee B)=\tau(A)+\tau(B)$. 则称 $\tau$ 为 $F(S)$ 的一个概率真度函数.

例 4.2 (i) 设 $\mu$ 是 $\Omega$ 上的 Borel 概率测度, 则由 (7) 式定义的 $\tau_{\mu}$ 是定义 4.1 意义下的概率真度 函数.

(ii) 设 $v \in \Omega$, 则 $v$ 也是定义 4.1 意义下的概率真度函数.

下面考察 $\tau$ 的基本性质.

命题 4.3 (i) 若 $\vdash A$, 则 $\tau(A)=1$.

(ii) 若 $A$ 与 $B$ 可证等价, 则 $\tau(A)=\tau(B)$.

(iii) $\tau(\neg A)=1-\tau(A)$.

(iv) 若 $A$ 是可驳公式 $\perp$, 则 $\tau(A)=0$.

(v) $\tau(A)+\tau(B)=\tau(A \wedge B)+\tau(A \vee B)$.

(vi) $\tau(A \leftrightarrow B)=\tau(A \rightarrow B)+\tau(B \rightarrow A)-1$.

(vii) $1+\tau(A \wedge B)=\tau(A)+\tau(A \rightarrow B)$.

(viii) $\tau(A)+\tau(A \rightarrow B)=\tau(B)+\tau(B \rightarrow A)$.

(ix) $\tau(B) \leqslant \tau(A \rightarrow B)$.

(x) $\tau(A \wedge B) \leqslant \min \{\tau(A), \tau(B)\} \leqslant \max \{\tau(A), \tau(B)\} \leqslant \tau(A \vee B)$.

(xi) $\tau(A \wedge B) \geqslant \tau(A)+\tau(B)-1$.

(xii) $\tau(B) \geqslant \tau(A)+\tau(A \rightarrow B)-1$.

(xiii) 若 $\tau(A \rightarrow B)=1$, 则 $\tau(A) \leqslant \tau(B)$.

(xiv) $\tau(A \rightarrow C) \geqslant \tau(A \rightarrow B)+\tau(B \rightarrow C)-1$.

证明以 (v) 为例. 由于 $A \sim A \wedge(B \vee \neg B) \sim(A \wedge B) \vee(A \wedge \neg B)$, 而 $A \wedge B$ 与 $A \wedge \neg B$ 是不相 容的, 所以 $\tau(A)=\tau((A \wedge B) \vee(A \wedge \neg B))=\tau(A \wedge B)+\tau(A \wedge \neg B)$. 又由于 $A \vee B$ 与 $(A \wedge \neg B) \vee B$ 可证等价以及 $A \wedge \neg B$ 与 $B$ 也不相容知 $\tau(A \vee B)=\tau((A \wedge \neg B) \vee B)=\tau(A \wedge \neg B)+\tau(B)$. 所以 $\tau(A)=\tau(A \wedge B)+\tau(A \wedge \neg B)=\tau(A \wedge B)+\tau(A \vee B)-\tau(B)$, 即得 $(\mathrm{v})$.

下面给出概率真度函数的等价刻画.

定理 4.4 设 $\tau: F(S) \rightarrow[0,1]$ 是映射, 则 $\tau$ 是 $F(S)$ 的概率真度函数 iff $\forall A, B \in F(S), \tau$ 满足

(i) 若 $\vdash A$, 则 $\tau(A)=1$.

(ii) 若 $\vdash \neg A$, 则 $\tau(A)=0$.

(iii) $\tau(A)+\tau(B)=\tau(A \vee B)+\tau(A \wedge B)$.

证明 由命题 4.3 中的 (i), (iv) 和 (v) 知必要性成立, 下证充分性. (K1) 与 (K2) 显然成立. 又 当 $A$ 与 $B$ 不相容时 $A \wedge B$ 为可驳公式, 所以由 (ii) 和 (iii) 知 (K4) 也成立, 因此只须证 (K3). 先证 $\tau(\neg A)=1-\tau(A)$. 事实上, 由 $\neg A \vee A$ 为定理, $\neg A \wedge A$ 为可驳公式及 (i) 和 (iii) 知 $1=\tau(\neg A \vee A)=$ 
$\tau(\neg A)+\tau(A)-\tau(\neg A \wedge A)=\tau(\neg A)+\tau(A)$. 所以 $\tau(\neg A)=1-\tau(A)$. 设 $\vdash A \rightarrow B$, 则 $\vdash \neg A \vee B$, 从而 由 (i) 及 (iii) 知 $1=\tau(\neg A \vee B)=\tau(\neg A)+\tau(B)-\tau(\neg A \wedge B)=1-\tau(A)+\tau(B)-\tau(\neg A \wedge B)$. 所以 $\tau(A) \leqslant \tau(B)$.

定理 4.5 设 $\tau: F(S) \rightarrow[0,1]$ 是映射, 则 $\tau$ 是 $F(S)$ 的概率真度函数 iff $\forall A, B \in F(S), \tau$ 满足

(i) 若 $A$ 是公理, 则 $\tau(A)=1$.

(ii) $\tau(\neg A)=1-\tau(A)$.

(iii) $\tau(A)+\tau(A \rightarrow B)=\tau(B)+\tau(B \rightarrow A)$.

证明 由命题 4.3 知, 也只需证充分性. 由 (iii) 知当 $\tau(A)=\tau(A \rightarrow B)=1$ 时必有 $\tau(B)=1$. 由 (i) 和这一事实可知对任一定理 $A, \tau(A)=1$. 设 $\vdash A \rightarrow B$, 则 $\tau(A \rightarrow B)=1$. 由 (iii) 知 $\tau(A)=$ $\tau(B)+\tau(B \rightarrow A)-\tau(A \rightarrow B)=\tau(B)+\tau(B \rightarrow A)-1 \leqslant \tau(B)$, 这就证得 (K3) 成立, 从而对于可证等 价的公式 $A, B$, 有 $\tau(A)=\tau(B)$. 下证 (K4). 由 (ii) 和 (iii) 知

$$
\begin{aligned}
\tau(A \vee B) & =\tau(\neg A \rightarrow B)=\tau(B \rightarrow \neg A)+\tau(B)-\tau(\neg A) \\
& =\tau(B)+\tau(A)-1+\tau(B \rightarrow \neg A)=\tau(B)+\tau(A)-1+\tau(\neg B \vee \neg A) \\
& =\tau(B)+\tau(A)-\tau(A \wedge B) .
\end{aligned}
$$

所以 $\tau(A \vee B)=\tau(A)+\tau(B)-\tau(A \wedge B)$. 当 $A$ 与 $B$ 不相容时 $\tau(A \wedge B)=0$, 从而 $\tau(A \vee B)=\tau(A)+\tau(B)$.

由定理 4.5 知本文定义的概率真度函数 $\tau$ 与文献 [24] 引入的语构真度函数 $\tau^{*}$ 等价, 但本文将证 明每个这样的 $\tau$ (或 $\left.\tau^{*}\right)$ 都可由 (7) 式表出. 下面再给出 $\tau$ 的一个等价刻画条件.

定理 4.6 设 $\tau: F(S) \rightarrow[0,1]$ 是映射, 则 $\tau$ 是 $F(S)$ 的概率真度函数 iff $\forall A, B \in F(S), \tau$ 满足

(i) 若 $\vdash A$, 则 $\tau(A)=1$.

(ii) 若 $\vdash \neg A$, 则 $\tau(A)=0$.

(iii) $\tau(A)+\tau(A \rightarrow B)=\tau(B)+\tau(B \rightarrow A)$.

证明 由命题 4.3 知只需证明充分性. 由定理 4.5 知只须证 $\forall A \in F(S), \tau(\neg A)=1-\tau(A)$. 由 (i) 和 (iii) 知若 $E$ 与 $F$ 可证等价, 则 $\tau(E)=\tau(F)$. 在 (iii) 中令 $B=\perp$ 为可驳公式, 则 $A \rightarrow \perp$ 与 $\neg A$ 可 证等价, $\perp \rightarrow A$ 与定理 $T$ 可证等价, 从而 $\tau(A)+\tau(\neg A)=\tau(A)+\tau(A \rightarrow \perp)=\tau(\perp)+\tau(\top)=0+1=1$. 所以定理 4.6 成立.

在证明 $F(S)$ 上的概率真度函数 $\tau$ 的表示定理之前, 再做些准备工作.

任取 $n \in N$ 及 $\left(x_{1}, \ldots, x_{n}\right) \in\{0,1\}^{n}$, 以 $\delta_{\left(x_{1}, \ldots, x_{n}\right)}$ 简记 (10) 式中的 $p_{1}^{x_{1}} \wedge \cdots \wedge p_{n}^{x_{n}}$, 即 $\delta_{\left(x_{1}, \ldots, x_{n}\right)}=$ $p_{1}^{x_{1}} \wedge \cdots \wedge p_{n}^{x_{n}}$.

定理 4.7 设 $\tau$ 是 $F(S)$ 的概率真度函数, $\left(x_{1}, \ldots, x_{n}\right) \in\{0,1\}^{n}$, 则

(i) $0 \leqslant \tau\left(\delta_{\left(x_{1}, \ldots, x_{n}\right)}\right) \leqslant 1$.

(ii) $\Sigma\left\{\tau\left(\delta_{\left(x_{1}, \ldots, x_{n}\right)}\right) \mid\left(x_{1}, \ldots, x_{n}\right) \in\{0,1\}^{n}\right\}=1$.

(iii) $\tau\left(\delta_{\left(x_{1}, \ldots, x_{n}, 0\right)}\right)+\tau\left(\delta_{\left(x_{1}, \ldots, x_{n}, 1\right)}\right)=\tau\left(\delta_{\left(x_{1}, \ldots, x_{n}\right)}\right)$.

证明 (i) 由 (K1) 知 (i) 成立.

(ii) 任取 $\left(x_{1}, \ldots, x_{n}\right),\left(y_{1}, \ldots, y_{n}\right) \in\{0,1\}^{n}$ 且 $\left(x_{1}, \ldots, x_{n}\right) \neq\left(y_{1}, \ldots, y_{n}\right)$, 则 $\delta_{\left(x_{1}, \ldots, x_{n}\right)}$ 与 $\delta_{\left(y_{1}, \ldots, y_{n}\right)}$ 不相容, 从而由 $(\mathrm{K} 4)$ 知 $\tau\left(\delta_{\left(x_{1}, \ldots, x_{n}\right)} \vee \delta_{\left(y_{1}, \ldots, y_{n}\right)}\right)=\tau\left(\delta_{\left(x_{1}, \ldots, x_{n}\right)}\right)+\tau\left(\delta_{\left(y_{1}, \ldots, y_{n}\right)}\right)$. 又, $\vee\left\{\delta_{\left(x_{1}, \ldots, x_{n}\right)} \mid\right.$ $\left.\left(x_{1}, \ldots, x_{n}\right) \in\{0,1\}^{n}\right\}$ 是定理, 所以 $\tau\left(\vee\left\{\delta_{\left(x_{1}, \ldots, x_{n}\right)} \mid\left(x_{1}, \ldots, x_{n}\right) \in\{0,1\}^{n}\right\}\right)=1$. 多次运用 (K4) 知 (ii) 成立.

(iii) 由 $\delta_{\left(x_{1}, \ldots, x_{m}, 0\right)} \vee \delta_{\left(x_{1}, \ldots, x_{m}, 1\right)}$ 与 $\delta_{\left(x_{1}, \ldots, x_{m}\right)}$ 可证等价, 以及 (ii) 便得 (iii). 
定理 4.8 设 $\tau$ 是 $F(S)$ 的概率真度函数, $A=A\left(p_{1}, \ldots, p_{n}\right) \in F(S)$, 则

$$
\tau(A)=\Sigma\left\{\tau\left(\delta_{\left(x_{1}, \ldots, x_{n}\right)}\right) \mid\left(x_{1}, \ldots, x_{n}\right) \in \bar{A}^{-1}(1)\right\} .
$$

证明 由命题 4.3(ii) 知, 若 $A$ 与 $B$ 可证等价, 则 $\tau(A)=\tau(B)$. 所以在不影响 $\tau(A)$ 的值时, 可设 $A$ 为析取范式 $A=\vee\left\{\delta_{\left(x_{1}, \ldots, x_{n}\right)} \mid\left(x_{1}, \ldots, x_{n}\right) \in \bar{A}^{-1}(1)\right\}$. 由定理 4.7 的证明知 $\tau(A)=\tau\left(\vee\left\{\delta_{\left(x_{1}, \ldots, x_{n}\right)} \mid\right.\right.$ $\left.\left.\left(x_{1}, \ldots, x_{n}\right) \in \bar{A}^{-1}(1)\right\}\right)=\Sigma\left\{\tau\left(\delta_{\left(x_{1}, \ldots, x_{n}\right)}\right) \mid\left(x_{1}, \ldots, x_{n}\right) \in \bar{A}^{-1}(1)\right\}$, 所以 (12) 式成立.

定理 4.9 (表示定理) 设 $\tau$ 是 $F(S)$ 的概率真度函数, 则存在 $\Omega$ 上的唯一 Borel 概率测度 $\mu$ 使 得对任一公式 $A \in F(S)$,

$$
\tau(A)=\mu\left(A^{-1}(1)\right) .
$$

证明 下面构造 $\mu$ 满足 (13) 式. 任取 $n \in N$, 令

$$
\mu\left(\left(x_{1}, \ldots, x_{n}\right) \times \prod_{m=n+1}^{\infty} X_{m}\right)=\tau\left(\delta_{\left(x_{1}, \ldots, x_{n}\right)}\right) .
$$

任取 $\Omega$ 的一拓扑闭集 $\Sigma$, 令

$$
\begin{gathered}
\mu\left(\Sigma(n) \times \prod_{m=n+1}^{\infty} X_{m}\right)=\Sigma\left\{\tau\left(\delta_{\left(x_{1}, \ldots, x_{n}\right)}\right) \mid\left(x_{1}, \ldots, x_{n}\right) \in \Sigma(n)\right\}, \\
\mu(\Sigma)=\lim _{n \rightarrow \infty} \mu\left(\Sigma(n) \times \prod_{m=n+1}^{\infty} X_{m}\right) .
\end{gathered}
$$

任取 $\Omega$ 的一 Borel 集 $\Delta \in \mathscr{B}(\Omega)$, 令

$$
\mu(\Delta)=\sup \{\mu(\Sigma) \mid \Sigma \subseteq \Delta \text { 为拓扑闭集 }\} .
$$

则由 (14)-(17) 式知 $\mu$ 是 $\Omega$ 上的 Borel 概率测度. 任取 $A \in F(S)$, 则由定理 4.8 知

$$
\begin{aligned}
\tau(A) & =\Sigma\left\{\tau\left(\delta_{\left(x_{1}, \ldots, x_{n}\right)}\right) \mid\left(x_{1}, \ldots, x_{n}\right) \in \bar{A}^{-1}(1)\right\} \\
& =\mu\left(\bar{A}^{-1}(1) \times \prod_{m=n+1}^{\infty} X_{m}\right)=\mu\left(A^{-1}(1)\right)
\end{aligned}
$$

即 (13) 式成立. 设 $\nu$ 是满足 (13) 式的 $\Omega$ 上的 Borel 概率测度, 则 $\nu$ 也满足 (14)-(17) 式, 所以由引理 3.21 知 $\mu=\nu$.

由定理 4.9 知在二值命题逻辑中只有形如 (7) 式的概率真度函数. 这也是本文在一开始就用拓扑 空间 $\Omega$ 上的 Borel 概率测度而不用一般概率测度来定义逻辑公式的概率真度的原因之一 (最重要的 原因在于用 Borel 概率测度定义的公式的概率真度可将概率逻辑学中公式的概率以及计量逻辑学中 公式的真度均可作为概率真度的特例而纳入到统一的框架中, 进而实现概率逻辑学与计量逻辑学的融 合与统一).

\section{5 结束语}

本文利用赋值空间 $\Omega$ 上的 Borel 概率测度在二值命题逻辑中引入了公式的概率真度的概念, 把 概率逻辑学中公式的概率以及计量逻辑学中公式的真度均作为概率真度的特例而纳入到了统一的框 
架中, 从而实现了概率逻辑学与计量逻辑学的融合和统一. 证明了概率真度函数与 $\Omega$ 上的 Borel 概率 测度是一样多的, 逻辑闭理论与 $\Omega$ 中的拓扑闭集是一一对应的. 本文第二部分又给出了概率真度函 数的公理化定义及其表示定理, 从而建立了二值命题逻辑框架下的 Borel 型概率计量逻辑的基本理论 体系.

利用概率真度函数 $\tau_{\mu}$ 可以像在计量逻辑学中那样引入公式间的相似度及伪度量, 建立相应的概 率逻辑度量空间并展开程度化推理 ${ }^{[11-13]}$. 同时, 基于 $\tau_{\mu}$ 还可引入逻辑理论的发散度及相容度概念 以解决逻辑理论的相容性问题.

另外, 本文的方法也可应用到多值命题逻辑中, 进而建立多值命题逻辑框架下的概率计量逻辑理 论, 我们将于另文中展开具体讨论.

\section{参考文献}

1 Hailperin T. Probability logic. Notre Dame J Form Logic, 1984, 25: 198-212

2 Nilsson N J. Probabilistic logic. Artif Intell, 1986, 28: 71-87

3 Adam E W. A Primer of Probability Logic. Stanford: CSLI Publications, 1998. 11-34

4 Hailperin T. Sentential Probability Logic. London: Associated University Press, 1996. 187-212

5 Wang G J, Fu L, Song J S. Theory of truth degrees of propositions in two-valued logic. Sci China Ser A-Math, 2002, 45: $1106-1116$

6 Wang G J, Leung Y. Integrated semantics and logic metric spaces. Fuzzy Set Syst, 2003, 136: 71-91

7 Li B J, Wang G J. Theory of truth degrees of formulas in Lukasiewicz n-valued propositional logic and a limit theorem. Sci China Ser F-Inf Sci, 2005, 48: 727-736

8 Li J, Wang G J. Theory of truth degrees of propositions in the logic system $\mathcal{L}_{n}^{*}$. Sci China Ser F-Inf Sci, 2006, 49: 471-483

9 Wang G J, Duan Q L. Theory of (n) truth degrees of formulas in modal logic and a consistency theorem. Sci China Ser F-Inf Sci, 2009, 52: 70-83

10 Zhou H J, Wang G J. A new theory consistency index based on deduction theorems in several logic systems. Fuzzy Set Syst, 2006, 157: 427-443

11 Zhou H J, Wang G J, Zhou W. Consistency degrees of theories and methods of graded reasoning in n-valued $R_{0}$-logic (NM-logic). Int J Approx Reason, 2006, 43: 117-132

12 Zhou H J, Wang G J. Generalized consistency degrees of theories w.r.t. formulas in several standard complete logic systems. Fuzzy Set Syst, 2006, 157: 2058-2073

13 Wang G J, Zhou H J. Quantitative logic. Inform Sciences, 2009, 179: 226-247

14 Wang G J, Zhou H J. Introduction to Mathematical Logic and Resolution Principle. Beijing/Oxford: Science Press and Alpha Science International Limited, 2009. 257-324

15 Wang G J, Hui X J. Randomization of classical inference patterns and its application. Sci China Ser F-Inf Sci, 2007, 50: $867-877$

16 Kolmogorov A N. Foundations of Probability. New York: Chelsea Publishing Co, 1950. 2-12

17 Hamilton A G. Logic for Mathematicians. London: Cambridge University Press, 1978. 27-36

18 Halmos P R. Measure Theory. New York: Springer, 1974. 154-160, 183

19 Cohn D L. Measure Theory. Boston: Birkhuser, 1980. 196-296

20 Munkres J R. Topology. 2nd ed. Beijing: China Machine Press, 2004. 119-125

21 Zhou H J, Wang G J. Characterizations of maximal consistent theories in the formal deductive system $\mathscr{L}^{*}$ (NM-logic) and Cantor space. Fuzzy Set Syst, 2007, 158: 2591-2604

22 Mill J V. Infinite-Dimensional Topology. Amsterdam: North-Holland, 1988. 17-136

23 王国俊, 王伟, 宋建社. 命题逻辑中极大和谐理论之集上的拓扑与 Cantor 三分集. 陕西师范大学学报 (自然科学版), 2007, 35: $1-5$

24 张东晓, 李立峰. 二值命题逻辑公式的语构程度化方法. 电子学报, 2008, 36: 325-330 


\title{
Borel probabilistic and quantitative logic
}

\author{
ZHOU HongJun* \& WANG GuoJun
}

College of Mathematics and Information Science, Shaanxi Normal University, Xi'an 710062, China

*E-mail: hjzhou@snnu.edu.cn, sdzhjun@gmail.com

\begin{abstract}
The present paper introduces the notion of the probabilistic truth degree of a formula by means of Borel probability measures on the set of all valuations, endowed with the usual product topology, in classical twovalued propositional logic. This approach not only overcomes the limitations of quantitative logic, which require the probability measures on the set of all valuations to be the countably infinite product of uniform probability measures, but also remedies the drawback that probability logic behaves only locally. It is proved that the notions of truth degree, random truth degree in quantitative logic and the probability of formulas in probability logic can all be brought as special cases into the unified framework of the probabilistic truth degree. Thus quantitative logic and probability logic are unified. It also proves a one-to-one correspondence between deductively closed theories and topologically closed subsets of the space of all valuations, and a one-to-one correspondence between probabilistic truth degree functions and Borel probability measures on the space of all valuations. The second part of the present paper proposes an axiomatic definition of the probabilistic truth degree, and it is finally proved that each probabilistic truth degree function is represented by a unique Borel probability measure on the space of all valuations in the way given in the first part. Thus a theory which we call probabilistic and quantitative logic in the framework of classical propositional logic is established.
\end{abstract}

Keywords probabilistic truth degree, finite separation property, probability logic, quantitative logic, probabilistic and quantitative logic 